\title{
Effects of Lipid Composition on Bilayer Membranes Quantified by All-Atom Molecular Dynamics
}

\author{
Wei Ding, Michail Palaiokostas, Wen Wang, and Mario Orsi* \\ School of Engineering \& Materials Science, Queen Mary University of London, Mile End Road, London, E1 4NS, U.K.
}

Supporting Information

ABSTRACT: Biological bilayer membranes typically contain varying amounts of lamellar and nonlamellar lipids. Lamellar lipids, such as dioleoylphosphatidylcholine (DOPC), are defined by their tendency to form the lamellar phase, ubiquitous in biology. Nonlamellar lipids, such as dioleoylphosphatidylethanolamine (DOPE), prefer instead to form nonlamellar phases, which are mostly nonbiological. However, nonlamellar lipids mix with lamellar lipids in biomembrane structures that remain overall lamellar. Importantly, changes in the lamellar vs nonlamellar lipid composition are believed to affect membrane function and modulate membrane proteins. In this work, we employ atomistic molecular dynamics simulations to quantify how a range of bilayer properties are altered by variations in the lamellar vs nonlamellar lipid composition. Specifically, we simulate five DOPC/ DOPE bilayers at mixing ratios of $1 / 0,3 / 1,1 / 1,1 / 3$, and $0 / 1$. We examine properties including lipid area and bilayer thickness, as well as the transmembrane profiles of electron density, lateral pressure, electric field, and dipole potential. While the bilayer structure is only marginally altered by lipid composition changes, dramatic effects are observed for the lateral pressure, electric field, and dipole potential profiles. Possible implications for membrane function are discussed.

\section{INTRODUCTION}

The lipid bilayer plays many key structural and functional roles within biological membranes. ${ }^{1}$ For example, it envelops cells, compartmentalizes the intracellular space, and acts as a selective barrier to permeation. The lipid bilayer also supports, and interacts with, numerous proteins. A detailed understanding of the properties of lipid bilayers is therefore central to biology, and is also relevant to many applications in the medical and pharmaceutical fields, ranging from biosensors ${ }^{2,3}$ to drug design and delivery. ${ }^{4-6}$ Unfortunately, the current knowledge on lipid membranes is limited, especially with respect to molecular-level properties and phenomena. Membrane properties can exhibit significant variations as a function of depth inside the bilayer, yet measuring experimentally such variations can be extremely difficult, because of the membrane's very small thickness $(\sim 5$ $\mathrm{nm}$ ) compounded by high heterogeneity, disorder, and fluidity. A different, complementary approach to experimental investigation is represented by molecular dynamics (MD) computer simulation. In particular, MD has proved to be a powerful tool to study many aspects of biological membranes at the nanoscale. $^{7-11}$

In this work, we apply atomistic MD simulations to investigate a fundamental yet elusive area of membrane research, that is, the effect of lipid composition changes on membrane physical properties. More specifically, we focus on the effect of changes in the content of lamellar vs nonlamellar lipids. It is well-known that biological membranes are composed of a wide variety of lipid species, whose relative amounts are dynamically regulated. ${ }^{1}$ The most prevalent lipid type observed in biological membranes is represented by glycerophospholipids, which can be further categorized into the lamellar or nonlamellar subtypes according to their inherent phase behavior. Dioleoylphosphatidylcholine (DOPC) and dioleoylphosphatidylethanolamine (DOPE) are two typical representatives of the lamellar and nonlamellar lipid families, respectively. Between DOPC and DOPE, there is a small yet crucial structural difference in the head groups, as illustrated in Figure 1a. Specifically, PC lipids comprise a choline group, characterized by three terminal methyl $\left(\mathrm{CH}_{3}\right)$ groups, whereas PE lipids comprise a smaller amine group (where hydrogen atoms replace the choline methyl groups of PC). Importantly, DOPC and DOPE exhibit substantially different phase behaviors. DOPC lipids preferentially self-assemble into the lamellar phase, which is the predominant phase observed in real biological membrane. By comparison, if DOPE lipids are dispersed in water at biological conditions, a nonlamellar (inverse hexagonal) phase will be formed, in which water aggregates in columns lined by the lipid headgroups. ${ }^{13}$ Despite such intrinsic tendency toward a phase that is mostly nonbiological, DOPE and other nonlamellar lipids are widespread in real biomembranes, where they mix with lamellar lipids to form phases which, importantly, are overall lamellar. It

Received: July 9, 2015

Revised: October 12, 2015

Published: November 11, 2015 
(a)
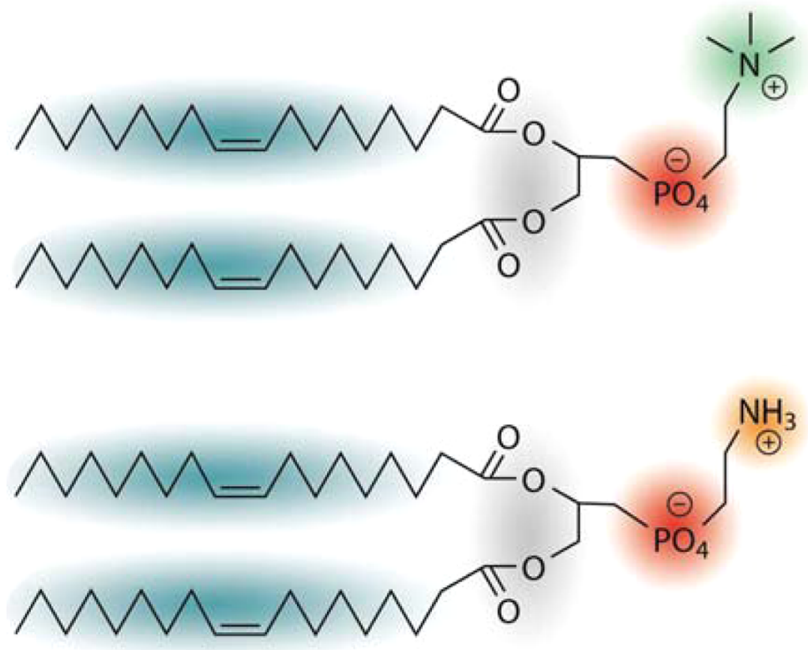

(b)

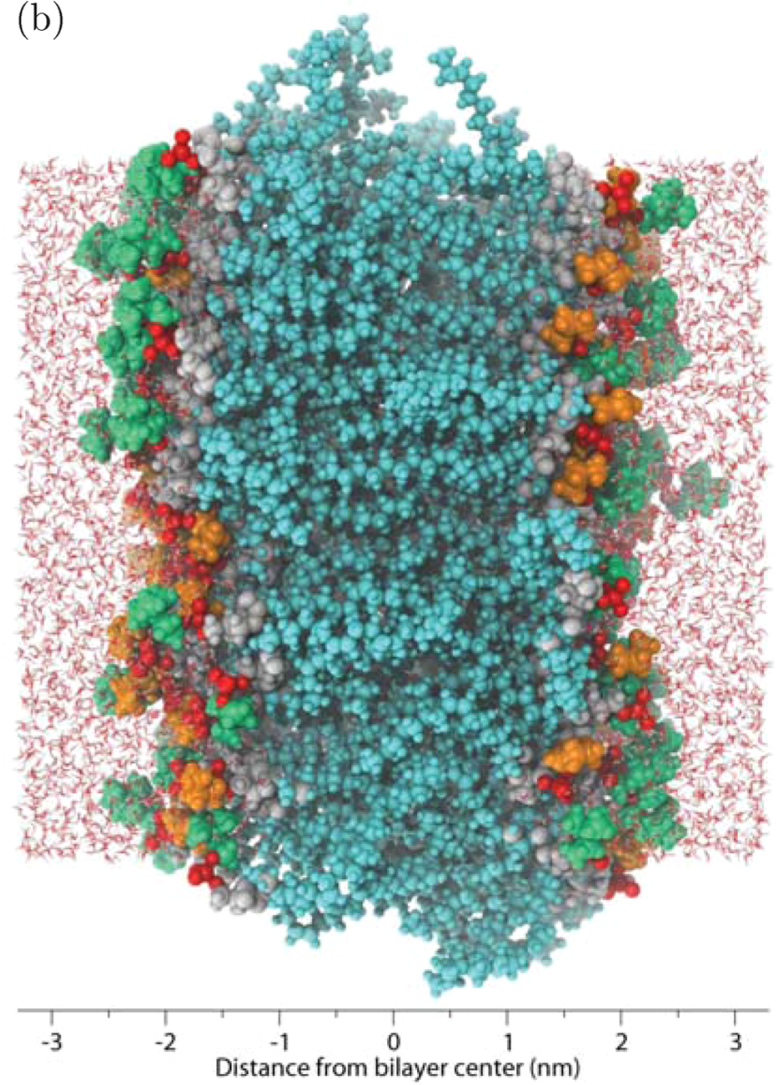

Figure 1. (a) Molecular structures of DOPC (above) and DOPE (below). Colors highlight hydrocarbon tails (cyan), glycerol-ester groups (gray), phosphate groups (red), choline (green) and ethanolamine (orange). (b) Representative snapshot from our simulation of the mixed DOPC/DOPE $\left(1 / 1\right.$ ratio) bilayer system (created using $\mathrm{VMD}^{12}$ ). Water molecules are depicted using a line representation, with oxygen and hydrogen colored red and white, respectively. Lipid molecules are represented using solid spheres, with molecular groups colored consistently with panel a.

may then be asked what is the role of nonlamellar lipids in vivo, and whether their frustrated desire to transition from lamellar to nonlamellar phases has any effects. A first intuitive explanation is that nonlamellar lipids are involved in membrane processes that require local nonlamellar structures (e.g., pores) to be formed, such as in cellular budding, fission or fusion. ${ }^{1,14}$ An additional hypothesis, less intuitive but potentially of great importance, points to the changes that nonlamellar lipids may induce on some key bilayer properties which will in turn control the behavior of the membrane and of any inclusions such as proteins. ${ }^{1,14-16}$ A significant and growing number of experimental studies have indeed provided evidence on how variations in the lamellar vs nonlamellar membrane lipid composition can control biological functions, such as protein folding, ${ }^{17}$ lipid biosynthesis, ${ }^{18}$ protein channel conductance ${ }^{19}$ and gating. ${ }^{15}$ However, a quantitative understanding of the mechanisms involved is hindered by experimental difficulties in accessing the relevant molecular-scale properties.

In this study, we perform atomistic molecular dynamics simulations to quantify relevant bilayer properties for mixed DOPC/DOPE systems at different relative compositions. In each bilayer studied, both leaflets contain the same amount of DOPC and DOPE lipids, that is, the lipid distribution is symmetric. It should be noted that lipid membranes in vivo can exhibit asymmetry of the lipid distribution across leaflets, and such asymmetry could have significant effects on the membrane's mechanical $^{20}$ and electrostatic properties. ${ }^{21}$ However, in this work the focus is exclusively on symmetric bilayers. We first consider basic membrane structural quantities, including lipid area, lipid volume, membrane thickness and electron density. These fundamental membrane properties have been previously investigated by experiments and other simulations, especially for pure DOPC bilayers. However, much fewer data have been previously reported regarding mixed DOPC/DOPE bilayers; in particular, to our knowledge, no previous all-atom simulation of mixed DOPC/DOPE bilayers has been reported. We will then focus on the transmembrane profiles of lateral pressure, dipole potential, and electric field; these properties are expected to vary significantly according to the position (depth) inside the membrane, and are hypothesized to play crucial roles in numerous membrane functions.

An inhomogeneous internal distribution of lateral pressure (or stress) can be predicted theoretically considering the different interactions that are expected to exist at different depths inside the lipid membrane. ${ }^{22}$ Experimentally, it has been possible to use molecular probes to detect intramembrane pressure changes. ${ }^{23}$ However, the results obtained are qualitative and limited to parts of the hydrocarbon chain region. Moreover, the probes used are relatively bulky, and can induce perturbations in the membrane as well as artifacts in the measurements. ${ }^{23,24}$ Despite current experimental limitations, theoretical considerations and data from molecular models indicate that the lateral pressure profile is characterized by depth-dependent pressure variations of the order of hundreds of atmospheres. ${ }^{22,24,25}$ Considerably large forces therefore act on proteins embedded in the membrane, as well as permeating molecules, and various hypotheses have been formulated 
regarding possible biological implications. ${ }^{1,5,14,15,26}$ For example, specific changes in the membrane lateral pressure may underpin the mechanism of general anesthesia by driving ion channels that conduct neural signals into closed states. ${ }^{27}$ A key factor that determines the shape and magnitude of the lateral pressure profile is represented by the specific composition of the lipid bilayer. Importantly, small changes in the lipid composition can induce large variations in the pressure profile. ${ }^{26}$ Previous simulation studies have focused on the effects of including cholesterol ${ }^{28}$ or changing unsaturation levels. ${ }^{29}$ Regarding the effect of systematic composition changes within lamellar/nonlamellar lipid mixtures, previous results have been obtained only from simulation of a simple coarsegrained model. ${ }^{30}$ Recently, Perrin et al. ${ }^{31}$ reported the pressure profile for a single composition (1/1) of a POPG (lamellar)/ POPE (nonlamellar) lipid mixture by employing the atomistic CHARMM36 lipid force field (as in our study). As far as we are aware, no previous atomistic MD study has been reported on pressure profiles from a range of compositions of lamellarnonlamellar mixed bilayers.

The dipole potential is an intramembrane electrostatic potential which originates from the preferential alignment of interfacial water dipoles and dipolar segments of the lipid molecules. An accurate measurement of the dipole potential would require nanoscale electrodes placed at different depths inside the bilayer; such an experiment has not been previously conducted, and has even been considered to be practically impossible. ${ }^{32}$ However, it has been possible to estimate the overall potential difference between the bilayer center and the outer water phase through indirect experimental measurements. In a seminal study on ion transport, the transbilayer conductivity of two fat-soluble ions with similar sizes were observed to be significantly different, with the permeability of the negative ion $\left(\mathrm{TPB}^{-}\right)$being $10^{5}$ times higher than that of the positive ion $\left(\mathrm{TPP}^{+}\right)$; this difference was ascribed to a positive electrostatic potential within the membrane. ${ }^{33}$ Subsequent studies, using various methods, confirmed the existence of such an electrical potential and estimated its magnitude to be several hundreds $m V^{32,34}$ It is important to point out that the dipole potential drops over a very short distance, corresponding to only 2-3 nm (which is the approximate thickness of each of the monolayers in a bilayer). Therefore, the corresponding electric field (which is the spatial derivative of the potential) can reach extremely large values, predicted to be in the range $10^{7}-10^{9} \mathrm{~V} / \mathrm{m}$. As a result, many electrostatically sensitive phenomena, such as the binding and permeation of charged or polar molecules, and the behavior of peptides and proteins, can be significantly affected by changes in the dipole potential. ${ }^{32,34}$ Previous studies have considered the effect of lipid composition changes on the dipole potential by focusing on the headgroup type, ${ }^{35}$ ether or ester linkage type, ${ }^{36}$ and double bonds in the chain region. ${ }^{37}$ Regarding lamellar/nonlamellar systems, previous investigations include a few experiments, ${ }^{35,38,39}$ a coarse-grained simulation study, ${ }^{30}$ but no atomistic simulations.

In the remainder of this paper, we report on a series of allatom $\mathrm{MD}$ simulations aimed at quantifying the effects of changes in the lamellar vs nonlamellar lipid content on membrane physical properties. It will be shown that the bilayer structure is relatively constant across the different systems, while the lateral pressure, dipole potential, and electric field profiles exhibit high sensitivity to composition.

\section{METHODS}

Bilayer Systems. Five bilayer systems were considered, comprising two pure DOPC and DOPE systems, and three mixed systems of varying DOPC/DOPE composition ratio. Table 1 details the composition of each system, and a snapshot

Table 1. Composition of the Simulated Bilayer Systems ${ }^{a}$

\begin{tabular}{lcccc}
\multicolumn{1}{c}{ system } & $N_{\text {DOPC }}$ & $N_{\text {DOPE }}$ & $N_{\text {water }}$ & $N_{\text {atoms }}$ \\
DOPC & 128 & 0 & 4300 & 30564 \\
DOPC/DOPE (3/1) & 96 & 32 & 4300 & 30276 \\
DOPC/DOPE (1/1) & 64 & 64 & 4300 & 29988 \\
DOPC/DOPE (1/3) & 32 & 96 & 4300 & 29700 \\
DOPE & 0 & 128 & 4300 & 29412
\end{tabular}

${ }^{a} N_{\text {DOPC }}$ and $N_{\text {DOPE }}$ represent the number of DOPC and DOPE molecules, respectively; $N_{\text {water }}$ indicates the number of water molecules; $N_{\text {atoms }}$ indicates the total number of atoms.

from a simulation of a representative mixed bilayer is shown in Figure 1b. All systems were fully hydrated with 4300 water molecules (water/lipid ratio $\sim 33.6) .^{40}$ It should be noted that under the studied conditions the pure DOPE system experimentally forms a nonlamellar (inverse hexagonal) phase. ${ }^{13}$ However, in our simulation the preassembled bilayer system maintains a lamellar structure, most likely because of the relatively small system size and the constraints imposed by the periodic boundary condition. While the simulated lamellar DOPE bilayer is thus rather artifactual, it will prove useful for providing a more complete and systematic data set, and to corroborate any observed composition-dependent trend. In fact, atomistic simulations of pure lamellar DOPE bilayers have also been used previously. ${ }^{41-43}$

It is nonetheless of interest to investigate whether the CHARMM36 force field can indeed predict the correct DOPE phase when the constraints of a preassembled system are removed. To this end, we have conducted a self-assembly test starting from a random solution of DOPE lipids and water, and we have been able to observe the formation of an inverse hexagonal phase, in agreement with the known experimental behavior. Full details of these simulations are reported in the Supporting Information.

Simulation Details. The starting structures of the bilayers were built using Packmol. ${ }^{44}$ Regarding the force field parameters, the CHARMM36 all-atom lipid force field ${ }^{45}$ and the CHARMM TIP3P (TIPS3P) water model ${ }^{46,47}$ were adopted. Molecular dynamics simulations were carried out using the LAMMPS program (version 11 Nov 2013). ${ }^{48,49}$ Simulation input files were generated using the charmm2lammps.pl tool available in the LAMMPS distribution and the psfgen plugin of VMD. ${ }^{12}$ The initial structures were energy minimized to remove potentially problematic close contacts between atoms. Each system was then simulated for a total of 1 $\mu$ s. The temperature was controlled at $303 \mathrm{~K}$ by applying the Langevin thermostat ${ }^{50}$ with a collision frequency of $1 \mathrm{ps}^{-1}$. The barostat by Berendsen et al. ${ }^{51}$ was used to maintain the system pressure at 1 atm, with a damping time of 1 ps and an isothermal compressibility of $4.6 \times 10^{-5} \mathrm{~atm}^{-1}$. The pressure was controlled semi-isotropically; that is, the $z$-component (perpendicular to the bilayer plane) of the pressure tensor was controlled independently from the other two (coupled) components along the $x$ - and $y$-axes (parallel to the bilayer plane). Periodic boundary conditions were applied in all 3 dimensions. The SHAKE algorithm was applied, with a relative 

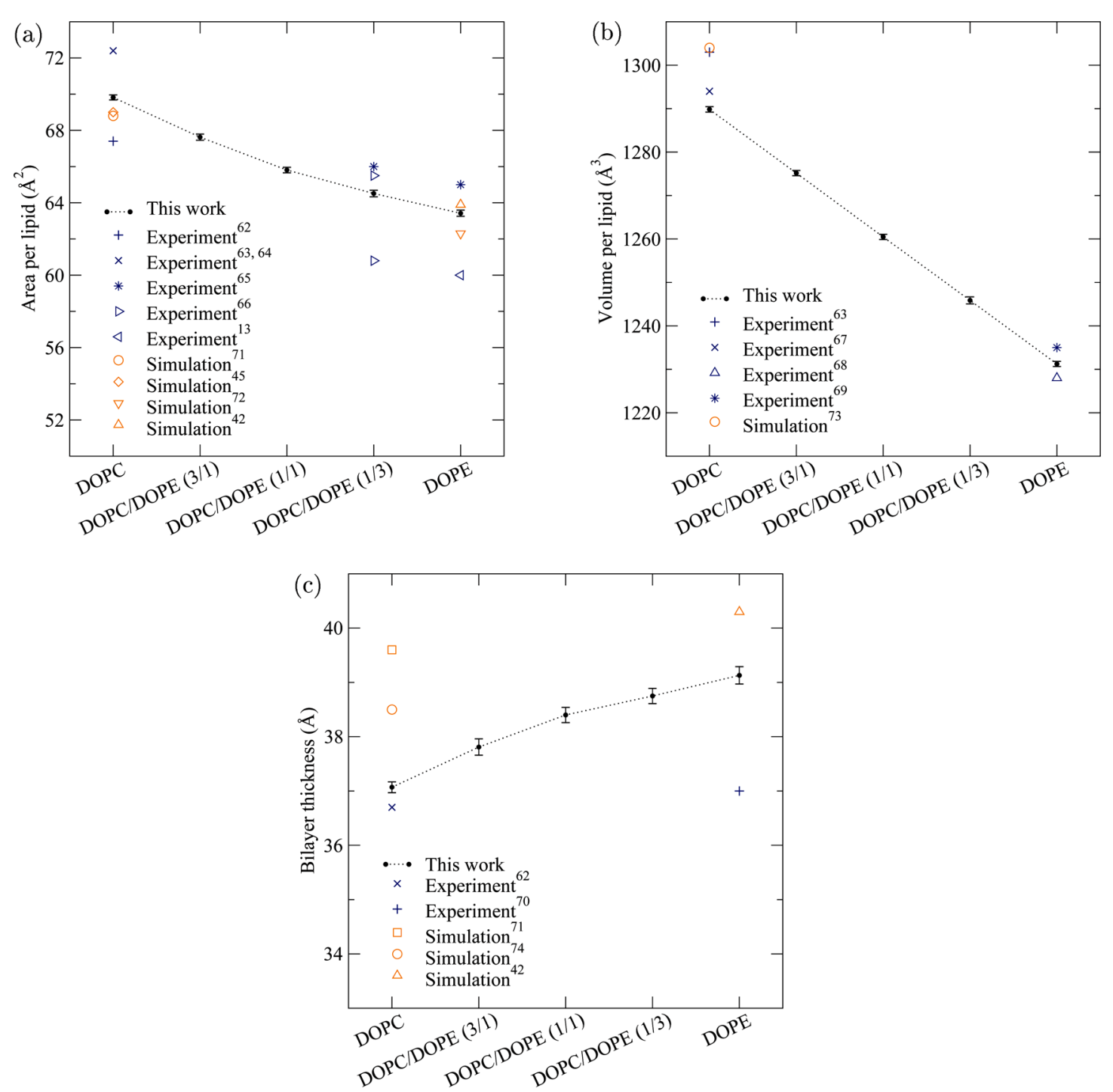

Figure 2. Structural properties: area per lipid (a), volume per lipid (b), and bilayer thickness (c). Error bars represent standard deviations. Literature data are superimposed from experiments (for $A_{\mathrm{L}}{ }^{13,62-66} V_{\mathrm{L}}^{63,67-69}$ and $d_{\mathrm{HH}}{ }^{62,70}$ ) and CHARMM 36 simulations (for $A_{\mathrm{L}}{ }^{42,45,71,72} V_{\mathrm{L}}{ }^{73}$ and $\left.d_{\mathrm{HH}}{ }^{42,71,74}\right)$. Note that not all the literature values were obtained at the same temperature as for this work (303 $\mathrm{K}$ ), but they are still in a comparable range $(275-318 \mathrm{~K})$, consistent with a fluid phase for the lipids considered.

tolerance of $10^{-5}$, to constrain all the bonds involving hydrogen atoms, as well as the $\mathrm{H}-\mathrm{O}-\mathrm{H}$ angle in water. The integration time step was $2 \mathrm{fs}$. At every step, the net momentum of the mass center of the entire system was removed to prevent any drifting. ${ }^{52}$ Nonbonded Lennard-Jones interactions were cut off beyond $10 \AA$, and the standard switching function in CHARMM was applied from 8 to $10 \AA^{53}$ Electrostatic interactions were computed using the PPPM (particle-particle particle-mesh) method, ${ }^{54}$ with a relative error tolerance of $10^{-5}$, and a real space cutoff of $10 \AA^{55}$ Intramolecular nonbonded interactions were considered according to the CHARMM convention, that is, interactions between atoms separated by less than three bonds (so-called 1-2 and 1-3 terms) were skipped, and 1-4 interactions were weighted according to the atoms types. ${ }^{53}$

Data Analysis Details. Data analysis was performed on the last $800 \mathrm{~ns}$ of each simulation, while the first $200 \mathrm{~ns}$ were regarded as equilibration. All targeted quantities were collected every 20 ps. Statistical uncertainties were estimated by the block averaging procedure, ${ }^{56,57}$ with a block size of $20 \mathrm{~ns}$. Error estimation analyses are shown in the Supporting Information. The transbilayer profiles were symmetrized with respect to the bilayer center by averaging over the two monolayers; unaveraged profiles can be found in the Supporting Information. Calculation details for specific properties are described in the following sections.

Structural Properties. The area per lipid, volume per lipid and bilayer thickness were calculated following previously reported protocols. ${ }^{9,58}$ The average area per lipid $\left(A_{\mathrm{L}}\right)$ is defined as the cross-section area of the whole system along the bilayer surface plane ( $x y$-plane) divided by the number of lipids in each monolayer (64 in our systems). The average volume per lipid is defined as $\left(V_{\text {box }}-V_{\text {water }}\right) / N_{\mathrm{L}}$, where $V_{\text {box }}$ is the volume of the whole simulation box, $V_{\text {water }}$ is the volume occupied by the water molecules, and $N_{\mathrm{L}}=128$ is the total number of lipids in our systems. The bilayer thickness $d_{\mathrm{HH}}$ is obtained from the peak to peak distance in the electron density profile.

Lateral Pressure Profile. To calculate the lateral pressure (or stress) distribution across the bilayer, the simulation box was 
first discretized into thin slabs parallel to the $x y$ plane. The thickness of each slab was set to $1 \AA$. For a slab centered at depth $z$, the lateral pressure profile $\Pi(z)$ is defined as ${ }^{24,30}$

$$
\Pi(z)=\frac{P_{x x}(z)+P_{y y}(z)}{2}-P_{z z}(z)
$$

where $P_{x x}(z), P_{y y}(z)$, and $P_{z z}(z)$ are the diagonal elements of the pressure tensor. Owing to the symmetry of the system with respect to the direction normal to the bilayer, the tangential pressures are expected to converge to identical values, that is, $P_{x x}(z)=P_{y y}(z)$, hence only either of the two is strictly needed for the calculation. However, in practice, both of them were calculated and the average of the two was used, thus improving the calculation precision. The normal component $P_{z z}(z)$ was assumed constant and equal to the external pressure $\left(P_{z z}(z)=1\right.$ atm) for all slabs; this condition, required by mechanical equilibrium, ${ }^{59}$ is also consistent with previous work. ${ }^{30,41}$ The contributions to the pressure in each slab from the various interactions were accumulated following the approach by Harasima, ${ }^{60}$ as in previous work. ${ }^{30,61}$

Dipole Potential and Electric Field Profiles. The dipole potential profile $\Psi(z)$ was calculated from the charge density distribution $\rho(z)$ along the direction normal to the bilayer plane. To obtain $\rho(z)$, the systems were again subdivided into 1 $\AA$ thick slabs, and the atomic (partial) charges were accumulated and averaged in each slab. The relation between $\Psi(z)$ and $\rho(z)$ is defined by Poisson's equation:

$$
\frac{\mathrm{d}^{2} \Psi(z)}{\mathrm{d} z^{2}}=-\frac{\rho(z)}{\varepsilon_{0}}
$$

so the dipole potential can be calculated as ${ }^{36,55,61}$

$$
\Psi(z)=-\frac{1}{\varepsilon_{0}} \int_{z_{0}}^{z} \int_{z_{0}}^{z^{\prime}} \rho\left(z^{\prime \prime}\right) \mathrm{d} z^{\prime \prime} \mathrm{d} z^{\prime}
$$

where the constant $\varepsilon_{0}$ is the electrostatic permittivity in vacuum, and $z_{0}$ is the reference position where the potential is set to zero, which in our calculations corresponds to the center of the water region. The electric field $E(z)$ projected on the $z$ direction can be obtained as the negative of the spatial derivative of the dipole potential, i.e., $E(z)=-\mathrm{d} \Psi(z) / \mathrm{d} z$.

\section{RESULTS AND DISCUSSION}

Structural Properties. The calculated area per lipid $\left(A_{\mathrm{L}}\right)$, volume per lipid $\left(V_{\mathrm{L}}\right)$ and the bilayer thickness $\left(d_{\mathrm{HH}}\right)$ of the simulated systems are shown in Figure 2, together with available literature data from experiments and from simulations of the same all-atom force field used in this work. Numerical values for $A_{\mathrm{L}}, V_{\mathrm{L}}$, and $d_{\mathrm{HH}}$ from our simulations are tabulated in the Supporting Information.

It can be seen from Figure $2 \mathrm{a}$ that the area per lipid $A_{\mathrm{L}}$ decreases with the presence of increasing amounts of DOPE; this trend is intuitive considering the smaller size of the DOPE headgroup with respect to DOPC (see also Figure 1a). However, it is important to note that, quantitatively, the effect is rather small. In fact, the net reduction in $A_{\mathrm{L}}$ from comparing the extreme cases of pure DOPC to pure DOPE is only 9\%. Figure $2 \mathrm{a}$ also shows that our results fall within the range of the various data previously reported in the literature.

Regarding the lipid volume $V_{\mathrm{L}}$, our results again show a decreasing trend in response to increasing the DOPE content (Figure 2b). The actual differences between the different systems are however very small, with a maximum volume decrease of $\sim 5 \%$ when comparing the pure DOPC and DOPE systems. Figure $2 \mathrm{~b}$ also shows that our value for pure DOPC is marginally smaller (to within 1\%) than the available literature data, while there is an almost exact match with experiments for pure DOPE.

The data obtained for the bilayer thickness $d_{\mathrm{HH}}$ are displayed in Figure 2c. It can be seen that $d_{\mathrm{HH}}$ systematically increases with addition of DOPE. Quantitatively, however, the changes in thickness corresponding to composition changes are very small, consistently with previous observations for $A_{\mathrm{L}}$ and $V_{\mathrm{L}}$. In fact, the difference between the pure DOPC and DOPE systems is only $\sim 5 \%$. A comparison with literature data highlights that all the CHARMM36 simulation results (including ours) overestimate the experimental measurements for the only two systems for which $d_{\mathrm{HH}}$ has been previously reported, i.e., pure DOPC and DOPE.

To characterize the relation between changes in the structural properties and corresponding changes in lipid composition, we performed a least-squares fitting analysis of the data from this work reported in Figure 2. The results obtained indicate that the change in the lipid volume follows a linear relation with a high level of significance, while for lipid area and bilayer thickness the relations are best described by quadratic functions, although the level of significance is somewhat low (detailed results of the fitting calculations can be found in the Supporting Information).

While the linearity of the lipid volume changes is intuitive, the nonlinearity for lipid area and thickness is not. However, the origin of such nonlinear behavior is unclear. In a previous investigation, de Vries et al. ${ }^{75}$ obtained a trend similar to ours for the $A_{\mathrm{L}}$ change with DOPC/DOPE composition, and they attributed this to the combined effect from headgroup size difference, electrostatic interactions, and hydrogen-bonding capability. However, it must be noted that the atomistic model used in that study ${ }^{75}$ was supplemented with a repulsive potential between the $\mathrm{H}$ atoms of the $\mathrm{PE}$ amine group and all other atoms, and it is not clear how this ad hoc modification may have influenced the trend reported.

Overall, experimental evidence on the effect of systematic composition changes appears necessary to validate the findings from simulations; unfortunately, however, measurements reported to date are rather scattered, as it can be seen from Figure 2.

The electron density profiles are shown in Figure 3. As expected from the previously reported structural results, the electron densities are characterized by very minor differences across the different compositions. In particular, with increasing DOPE content the peaks in the headgroup region shift slightly out toward the water phase (consistently with the previously observed increase in $d_{\mathrm{HH}}$ ), by a maximum of $0.1 \mathrm{~nm}$ when comparing pure DOPC and DOPE. The peak magnitudes are effectively unchanged across the different systems, while the central minimum decreases marginally, by at most $\sim 0.01 \mathrm{e}$ $\mathrm{nm}^{-3}$ when comparing pure DOPC to DOPE.

Lateral Pressure Profile. The lateral pressure profiles $\Pi(z)$ for the bilayer systems investigated are shown in Figure 4. In general, it can be noted that all the profiles display similar overall qualitative features. Upon entering the bilayer from the outer water phase, the lateral pressure rises sharply and forms large positive peaks located roughly at the interface between the water and lipid heads regions. Positive values of the lateral pressure profile generally indicate repulsive forces, acting to 


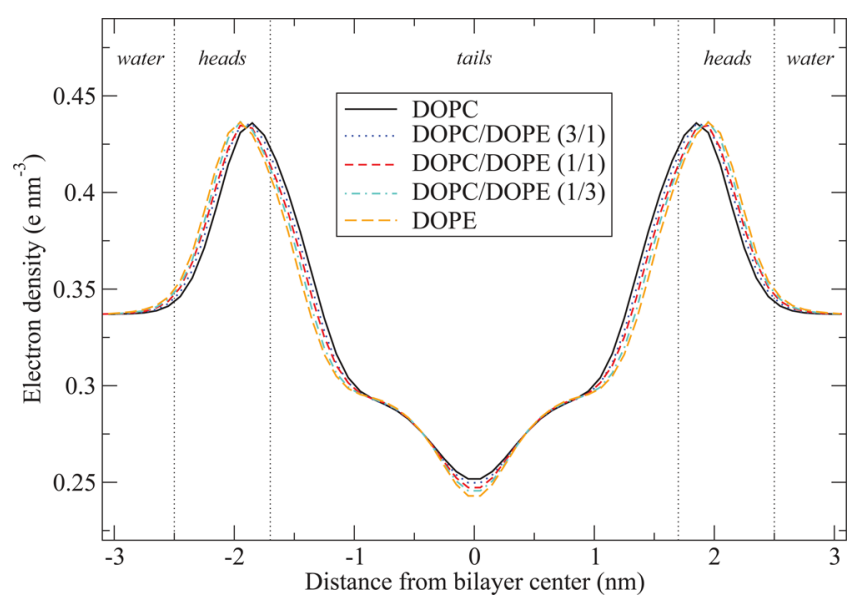

Figure 3. Electron density profiles. Vertical dotted lines indicate approximate boundaries between regions occupied predominantly by water, lipid heads, and hydrocarbon tails.

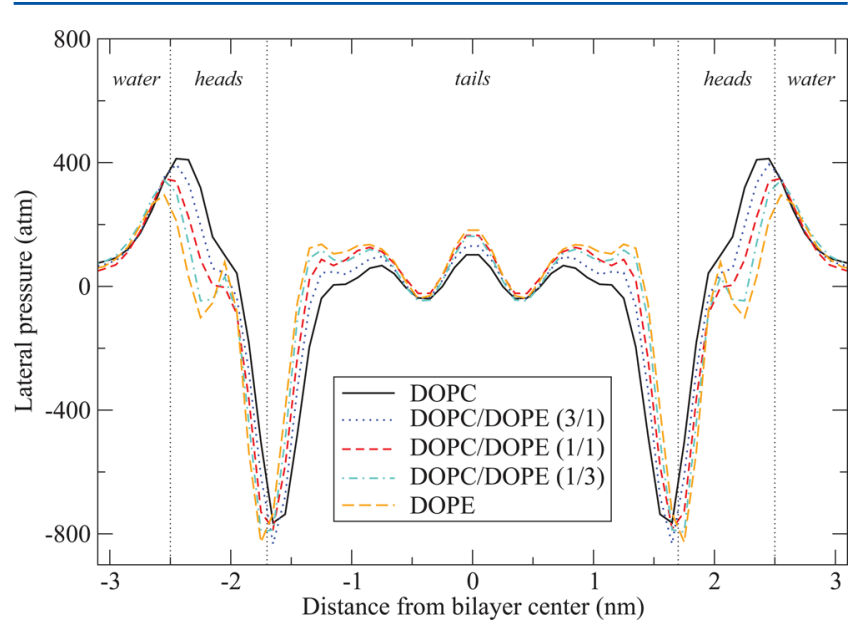

Figure 4. Lateral pressure profiles $(\Pi(z))$. Vertical dotted lines indicate approximate boundaries between regions occupied predominantly by water, lipid heads, and hydrocarbon tails.

enlarge the area of the bilayer. At the water-heads interface, a net repulsion is obtained from intermolecular contributions of steric, electrostatic, and hydration nature. ${ }^{22}$ Upon moving deeper inside the bilayer, the lateral pressure drops drastically, forming deep troughs approximately at the interface between the lipid heads and tails regions. In general, negative pressures correspond to the presence of intermolecular attractive forces, that act to reduce the bilayer area. In fact, this location corresponds to an interface involving a hydrophobic environment (the hydrocarbon tail region) with a strong tendency to reduce and minimize its area in contact with a hydrophilic environment (comprising water and lipid heads). The lateral pressure troughs can thus be seen as a reflection of the hydrophobic effect, whereby hydrophobic molecules cluster together in order to restrict their exposure to water. In the central part of the profiles, corresponding to the hydrocarbon core of the bilayers, the lateral pressure is mostly positive, comprising three peak regions with two corresponding local minima in between. The repulsive forces giving rise to the tails pressure are typically explained in terms of entropy losses. ${ }^{26}$ In particular, the tight molecular packing in the bilayer core forces the lipid tails to stretch (therefore losing entropy compared to isolated "free" tails), ultimately causing substantial tail-tail repulsion. $^{76,77}$

While all the profiles are similar qualitatively, remarkable quantitative differences and trends can be observed and related to changes in the lipid composition. Regarding the peaks at the interface between the water and lipid heads regions, corresponding to a distance of about $2.5 \mathrm{~nm}$ from the bilayer center, a top pressure of $\sim 410 \mathrm{~atm}$ is observed for the pure DOPC system. With the presence of increasing ratios of DOPE in the mixed systems, the peak pressure systematically decreases, reaching its lowest value for the pure DOPE system, at $\sim 290 \mathrm{~atm}$. The presence of growing amounts of DOPE also causes the main peaks to shift slightly outward, toward the water region; this correlates with the small increase in bilayer thickness noted earlier (Figures $2 \mathrm{c}$ and 3 ). For the two systems with highest DOPE content, i.e., DOPC/DOPE (1/3) and pure DOPE, the emergence of a pair of local peaks and troughs can also be observed in the middle of the lipid heads region. The local troughs, at $2.25 \mathrm{~nm}$ from the bilayer center, markedly enhance the pressure drops at the same location compared to DOPC; such drops amount to 2350 atm for DOPC/DOPE $(1 / 3)$ and $\sim 400$ atm for pure DOPE. Regarding the main
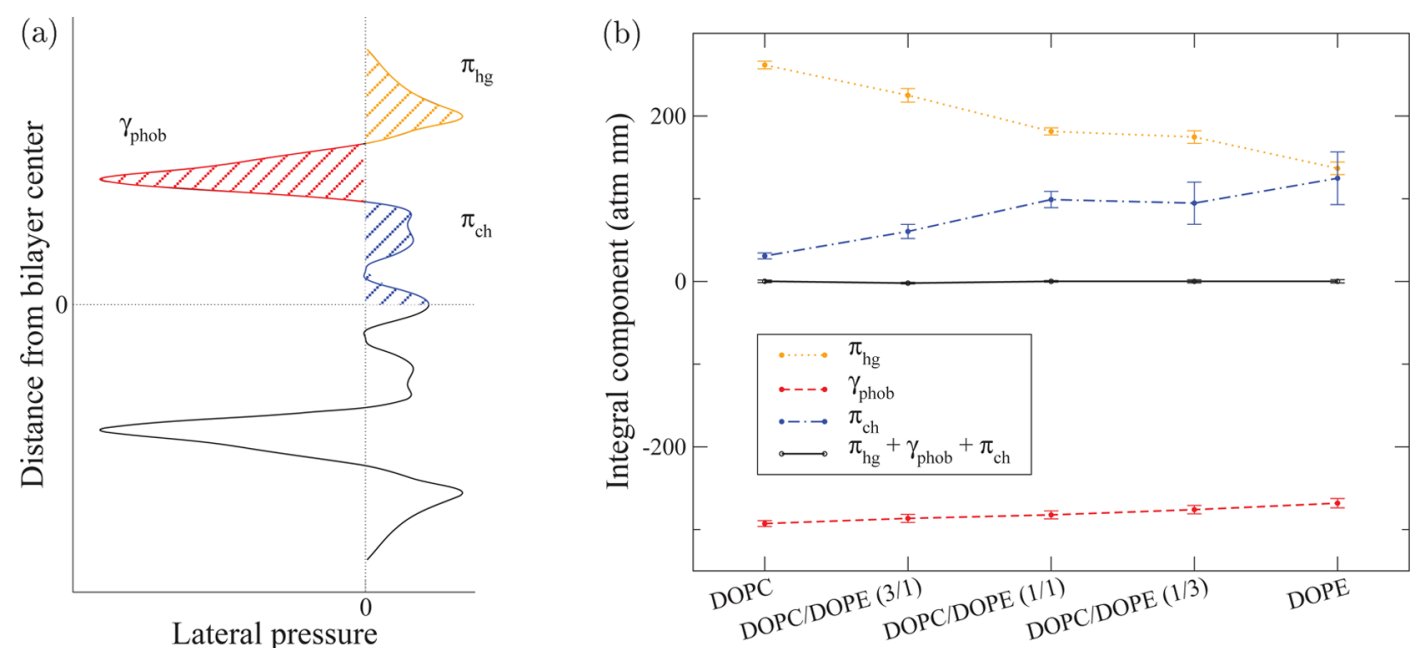

Figure 5. Integrals of different sections of the lateral pressure profile. (a) Schematic illustration of the quantified contributions. (b) Results obtained (error bars represent standard deviations). 
troughs, located at a distance of $\sim 1.7 \mathrm{~nm}$ from the bilayer center, it can be seen that the minima in the pressure fall in a relatively narrow range between $\sim-760$ and $\sim-830 \mathrm{~atm}$, without any systematic trend related to the DOPC/DOPE ratio. Composition-dependent effects are again apparent in the hydrocarbon tail core of the bilayers, where increasing the DOPE ratio systematically increases the lateral pressure, up to $80 \mathrm{~atm}$ for the central peak, and up to $\sim 100$ atm toward the top end of the tails (at $\sim 1 \mathrm{~nm}$ from the bilayer center), when comparing pure DOPC and DOPE.

To conduct a further quantitative assessment of the effect of the lipid composition on the pressure profile, we carried out an analysis in terms of the main "integrated contributions". ${ }^{14}$ In particular, we first subdivided the profile into three main sections (Figure 5a): (i) the headgroup ("hg") section corresponding to the part of the profile characterized by the positive pressure in the water and lipid heads regions; (ii) the hydrophobic ("phob") section characterized by the negative pressure corresponding to the main troughs; (iii) the chains ("ch") section corresponding to the positive pressure in the hydrocarbon tails (or chains) region. For each of these sections, we calculated the integrals of the lateral pressure profiles, as illustrated in Figure 5a. By integrating the pressure from key regions of the profiles, it is possible to capture the combined effect of peak magnitudes and peak widths, as obviously the integrals correspond to the areas under the curve for the related sections. The composition-dependent results obtained for the different bilayer systems are plotted in Figure 5b. First of all, it can be verified that, irrespective of the lipid composition, the sum over the three components is always zero, meaning that the integrals of the individual sections balance out, as required by mechanical equilibrium. Regarding the headgroup integral $\pi_{\mathrm{hg}}$, it can be seen that it decreases together with the presence of increasing amounts of DOPE, a trend which is consistent with the previously observed decrease in peak pressures in the same region (Figure 4 ). In particular, $\pi_{\mathrm{hg}}$ decreases by almost $50 \%$ from the pure DOPC system to pure DOPE. The trend observed for $\pi_{\mathrm{hg}}$ is countered by the chains integral $\pi_{\mathrm{ch}}$, which displays an enormous increase in relative terms, of approximately $400 \%$, from the pure DOPC to the pure DOPE system as a result of DOPE addition. Since it has been estimated that even a $10 \%$ change in the chains pressure can induce a very large shift in the conformational equilibrium of membrane proteins, ${ }^{26}$ our results suggest that even small shifts in the DOPC/DOPE composition can have significant biological repercussions. Incidentally, it has been proposed $^{14}$ that the relative size of the headgroup and chain contributions to the lateral pressure leads to the relation $\pi_{\mathrm{hg}} / \pi_{\mathrm{ch}} \approx 1$, which is consistent with the value of $1.1 \pm 0.3$ from our data. The hydrophobic integral $\gamma_{\text {phob }}$ represents the interfacial tension acting at the polar-apolar interface. ${ }^{14}$ It can be seen that the absolute value of $\gamma_{\text {phob }}$ decreases slightly with increasing DOPE ratio; in particular, we observe an overall reduction of $\sim 9 \%$ from pure DOPC to pure DOPE. Such a reduction in interfacial tension reflects the slight decrease in the polarapolar interfacial area observed previously (Figure 2a).

Dipole Potential Profile. The dipole potential profiles $\Psi(z)$ are displayed in Figure 6. It can be noticed that all the profiles share similar qualitative features, irrespective of the different lipid compositions. However, it is clear that there are substantial composition-dependent differences in the magnitude of the potentials. In particular, an evident trend can be observed, whereby increasing amounts of DOPE induce

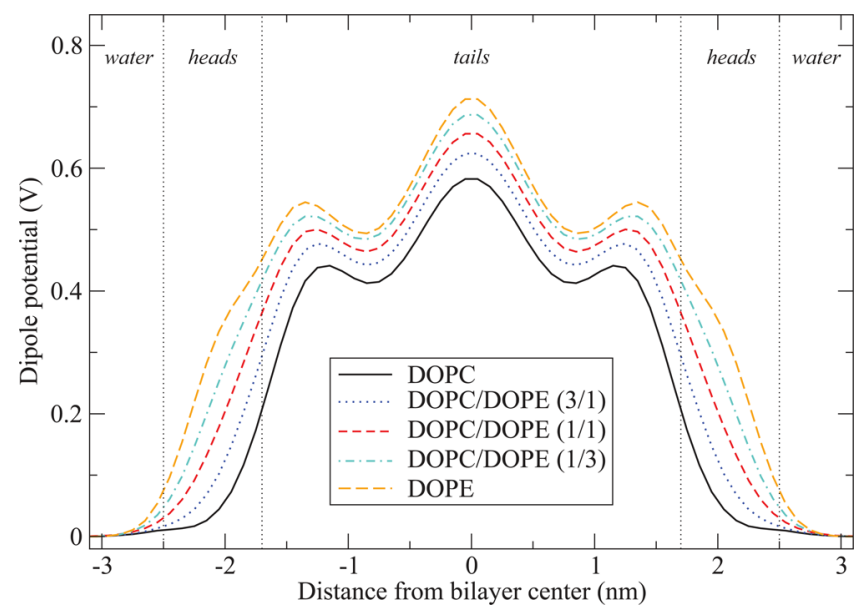

Figure 6. Dipole potential profiles $\Psi(z)$. Vertical dotted lines indicate approximate boundaries between regions occupied predominantly by water, lipid heads, and hydrocarbon tails.

increasingly larger values of $\Psi(z)$ across the entire profile. Starting from the reference value of $0 \mathrm{mV}$ in the water phase, $\Psi(z)$ rises sharply across the lipid heads region, up to peak values from $441 \pm 26 \mathrm{mV}$ for DOPC to $545 \pm 24 \mathrm{mV}$ for DOPE, with the mixed systems displaying values distributed in between. These peaks are located at $\sim 1.3 \mathrm{~nm}$ from the bilayer center, corresponding to the top part of the lipid tails, near the glycerol-ester groups. The profiles can then be observed to drop and form local minima at $\sim 0.8 \mathrm{~nm}$ from the bilayer center, a region roughly corresponding to the double bond midway along the hydrocarbon tails. In the bilayer center, the dipole potential reaches a global maximum with values ranging from $582 \pm 28 \mathrm{mV}$ for DOPC to $712 \pm 25 \mathrm{mV}$ for DOPE, corresponding to an increase of $22 \%$.

The profile obtained for the pure DOPC bilayer is consistent with the profile reported by Warshaviak et al. ${ }^{74}$ from simulation of DOPC with the same force field used in our work (CHARMM36), as expected. Regarding the mixed DOPC/ DOPE bilayers, we are not aware of any previous dipole potential data from either atomistic simulations or experiments. There are however some experimental studies on singlecomponent bilayers. Specifically, earlier measurements by Pickar and Benz ${ }^{38}$ indicated values of 0.224 and $0.215 \mathrm{~V}$ for DOPC and DOPE bilayers, respectively. These results have been more recently amended by Schamberger and Clarke ${ }^{39}$ to include a more accurate estimate of hydration energies, yielding updated values of $0.343 \mathrm{~V}$ for DOPC and $0.334 \mathrm{~V}$ for DOPE. ${ }^{39}$ Regardless of the correction, both sets of data indicate a (marginally) larger potential for DOPC compared to DOPE, which is in contrast to the trend observed in our simulation results. However, more recent data by Starke-Peterkovic and Clarke ${ }^{35}$ showed an increase in the dipole potential, from 0.410 to $0.461 \mathrm{~V}$, when comparing a pure DMPC system to an equimolar mixture of DMPC and DMPE, in qualitative agreement with our results (note that DMPC/DMPE and DOPC/DOPE are characterized by differences which are small and localized in the hydrocarbon region, thus it is acceptable to make a qualitative comparison in relation to their dipole potential). In any case, it is not clear how reliable the experimental estimates are, given the well-known difficulties in measuring the dipole potential, and especially in relation to ranking different values from $\mathrm{PC}$ vs $\mathrm{PE}$ systems. In fact, all three reported $\mathrm{PC} / \mathrm{PE}$ pairs of experimental values are very close to 
each other, with a level of uncertainty that in one case ${ }^{35}$ has been reported to be much larger $(\sim 0.15 \mathrm{~V})$ than the actual difference in $\mathrm{PC}$ vs $\mathrm{PE}$ values $(0.05 \mathrm{~V})$. No uncertainty estimates were reported for the other data, ${ }^{38,39}$ but the differences in PC vs PE values are so small $(0.009 \mathrm{~V}$ in both cases) that they are very much likely to be smaller than the uncertainty in the measurements.

In general, it should be noted that the experimental estimates for the dipole potential tend to be markedly lower than the simulation values. This is a well-known issue, consistently observed in previous atomistic simulations of lipid bilayers. $^{36,45,74,78-80}$ The discrepancy with respect to the experimental values has been ascribed, at least in part, to the lack of electronic polarizability in standard atomistic force fields. $^{80}$

To further investigate the molecular origin of $\Psi(z)$, as well as the effect of changing the lamellar-nonlamellar lipid ratio, we isolated the two separate contributions to $\Psi(z)$ from the water and lipid molecules. Results for such individual components are plotted in Figure 7, together with the net profiles (already

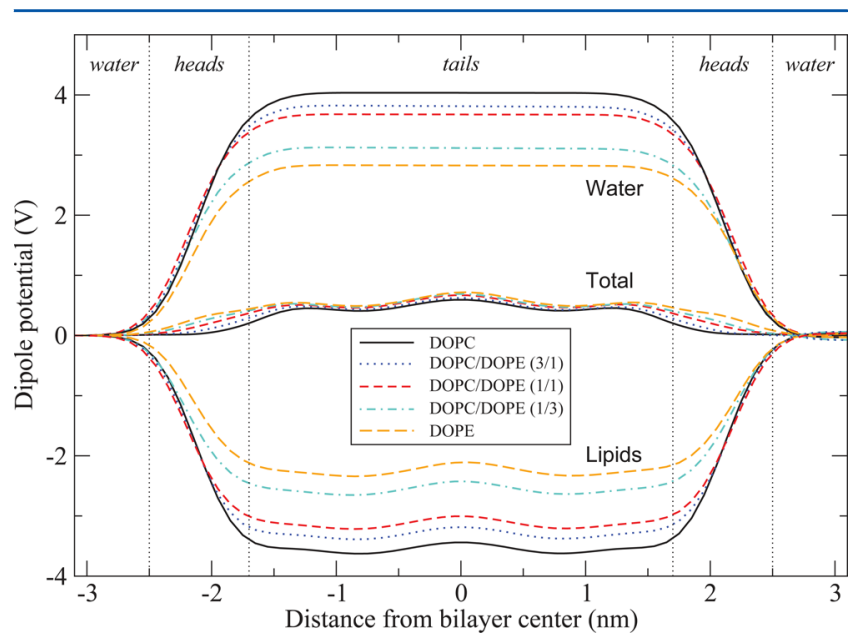

Figure 7. Individual contributions of the dipole potential profile from water and lipid molecules. The total net potentials are also plotted for reference (see Figure 6 for dedicated plot). Vertical dotted lines indicate approximate boundaries between regions occupied predominantly by water, lipid heads, and hydrocarbon tails.

reported in Figure 6) for reference. For all systems, it can be seen that the total net potential results from a competition between much larger positive and negative contributions due to water and lipids, respectively. This is generally consistent with previously reported data from atomistic simulations of pure DOPC systems. ${ }^{74,79}$ In the only previously reported simulation study on DOPC/DOPE mixed bilayers, the lipid dipoles (rather than water) were the main contributors to the total potential, and composition-related trends were also different from those observed here. ${ }^{30}$ However, that study ${ }^{30}$ employed a coarse-grained model which treats water molecules as single point dipoles without explicit hydrogen bonding capabilities, ${ }^{81,82}$ and hence the preferential alignment of water in the heads region (which determines the electric field) may not be accurately reproduced. The atomistic simulations reported here allow new quantitative insights to be obtained into composition-dependent effects. Specifically, Figure 7 shows that while the water contribution determines the positive sign of the overall net profiles, their composition-dependent trend is dictated by the lipid contributions. In fact, the observed increase in the overall net dipole potential brought about by the presence of increasing amounts of DOPE is determined by the lipid relative contributions overcompensating the water contributions (which instead display decreasing potential values with increasing DOPE ratios).

Electric Field. The projection of the electric field along the direction normal to the bilayer plane is reported in Figure 8 .

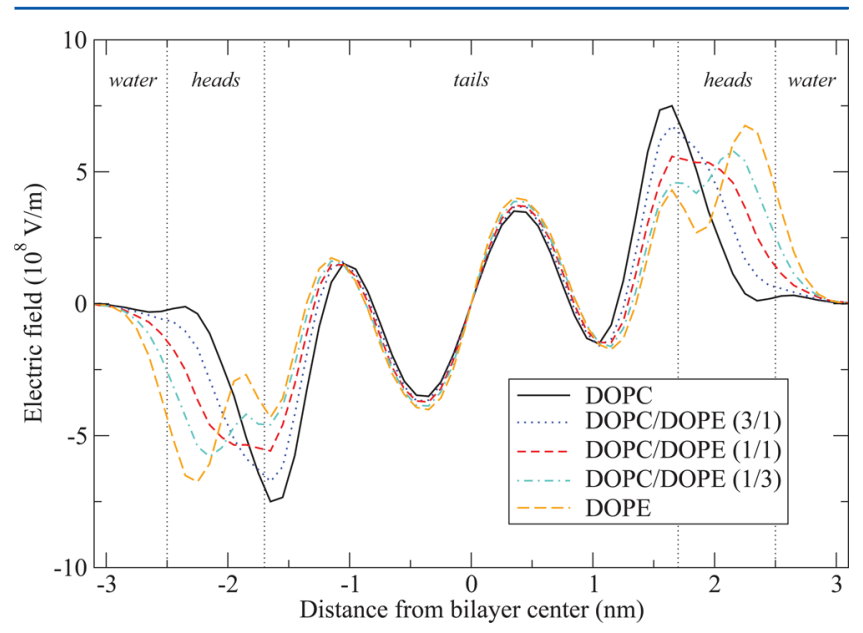

Figure 8. Electric field $E(z)$. Vertical dotted lines indicate approximate boundaries between regions occupied predominantly by water, lipid heads, and hydrocarbon tails.

Note that the curves are antisymmetrical, due to the vectorial nature of the electric field and the symmetry of the bilayer systems with respect to the central plane. Thus, field vectors pointing outward from the bilayer toward the water phase are indicated by positive values of $E(z)$ on the right-hand side of the diagram, and by corresponding negative values, of equal magnitude and equidistant from the origin, on the left-hand side (and vice versa for inward pointing vectors). In other words, the field profiles would be symmetric if calculated with respect to the normal for each leaflet. It can be seen that the electric field magnitude reaches huge peak values, with overall maxima of up to $\sim 7.5 \times 10^{8} \mathrm{~V} / \mathrm{m}$, located near or inside the lipid heads region depending on the system. Despite the absence of direct experimental measurements for comparison, such strong electric fields are consistent with the dipole potential profile, in relation to its peak magnitudes and steep gradients. ${ }^{32}$ In fact, the electric field strength corresponds to the spatial derivative of the dipole potential, which varies substantially over extremely small distances (see Figure 6). For pure DOPC, our result matches as expected a previously reported profile ${ }^{74}$ obtained from simulations of the same force field (CHARMM36). For the mixed systems, we are not aware of any previously reported $E(z)$ data from either simulation or experiment.

It is clear from Figure 8 that changes in the DOPC/DOPE ratio induce substantial redistributions of the electric field, especially corresponding to the lipid heads region and at the heads-tails interface. Regarding the pure DOPC bilayer, the largest peak magnitude corresponds to $\sim 7.5 \times 10^{8} \mathrm{~V} / \mathrm{m}$, and is located at the interface between the heads and tails regions as denoted on Figure 8. Considering the DOPC/DOPE (3/1) systems, the magnitude drops by $\sim 0.8 \times 10^{8} \mathrm{~V} / \mathrm{m}$, or $11 \%$. An even larger decrease of $\sim 1.1 \times 10^{8} \mathrm{~V} / \mathrm{m}$ in the field strength can be observed by comparing the DOPC/DOPE (3/1) and DOPC/DOPE (1/1) bilayers. The magnitude of the heads-tails 
interfacial peak decreases further until a value of $\sim 4.4 \times 10^{8} \mathrm{~V} /$ $\mathrm{m}$ for the pure DOPE bilayer, corresponding to an overall drop of $41 \%$ compared to the pure DOPC system. Interestingly, the two systems with highest DOPE content (DOPC/DOPE (1/3) and pure DOPE) are also characterized by additional features; in particular, their points of maximum field strength correspond to extra peaks (compared to the other systems) inside the heads region. In fact, taking into account all the peaks of maximum magnitude for each of the five systems, from pure DOPC to pure DOPE, such peaks can also be interpreted as shifting outward from the bilayer interior toward the water region, by as much as $0.6 \mathrm{~nm}$ when comparing the pure DOPC and DOPE bilayers. The largest composition-dependent effect on the field magnitude can be observed at a distance of $\sim 2.3$ $\mathrm{nm}$ from the bilayer center, where the field strength is almost zero for DOPC, while it grows dramatically for the mixed bilayers, up to $\sim 7 \times 10^{8} \mathrm{~V} / \mathrm{m}$ for DOPE. Overall, such $E(z)$ changes are expected to have significant effects on any charged or polar molecule interacting with the bilayer (e.g., permeants), as well as embedded peptides and proteins. Specifically, it was estimated that a change in field magnitude of $\sim 2 \times 10^{8} \mathrm{~V} / \mathrm{m}$ can already induce substantial shifts in protein conformational equilibria. $^{74}$ Charged protein residues are predicted to be especially sensitive, but also uncharged residues will be affected through interactions with the large dipole (3.5 D) that characterizes the peptide bond.

In the hydrocarbon tails core, the composition-dependent changes in $E(z)$ are more limited, as intuitively expected considering that there are no chemical differences between DOPC and DOPE tails. However, some effects can still be noted. In particular, the innermost peaks, located at $\sim 0.4 \mathrm{~nm}$ from the bilayer center, show increasing magnitudes with addition of DOPE, up to $0.5 \times 10^{8} \mathrm{~V} / \mathrm{m}$ when comparing pure DOPC with pure DOPE. Regarding the peaks at $\sim 1 \mathrm{~nm}$ from the bilayer center, a slight increase (up to $0.25 \times 10^{8} \mathrm{~V} / \mathrm{m}$ ) in magnitude is accompanied by a small shift outward (up to $\sim 0.1$ $\mathrm{nm}$ ) upon DOPE addition.

To further assess the biological relevance of the results obtained, it is useful to compare them to the electric fields corresponding to two other membrane-related potentials, i.e., the transmembrane potential and the surface potential, for which more knowledge exists compared to the dipole potential, because they are comparatively easier to study experimentally. ${ }^{32}$ The transmembrane potential arises from the different ion concentrations typically present between the inner and outer sides of biological membranes, and underlies the opening and closing of ion channels that control the transmission of electrical signals along neurons and muscle cells. The surface potential arises from charged lipid headgroups and polar molecules accumulating at the membrane surface, and controls the ion distribution at the cell surface. The electric field magnitudes related to the transmembrane and surface potentials have been estimated to be, respectively, $\sim 0.25 \times$ $10^{8} \mathrm{~V} / \mathrm{m}$ and $\sim 0.1 \times 10^{8} \mathrm{~V} / \mathrm{m}^{83}$ Considering that these field strengths are known to be responsible for significant biological activity, the composition-dependent effects for the dipole potential highlighted here, especially around the heads region, can be expected to also have major biological consequences. In fact, even the smaller DOPC/DOPE composition-dependent effects on $E(z)$ that we observed in the tails region are of comparable size to the field magnitudes from the transmembrane and surface potentials.
To further analyze the electric field, we also calculated the separate contributions from the water and lipid molecules, respectively (Figure 9). By comparing parts a and b of Figure 9,
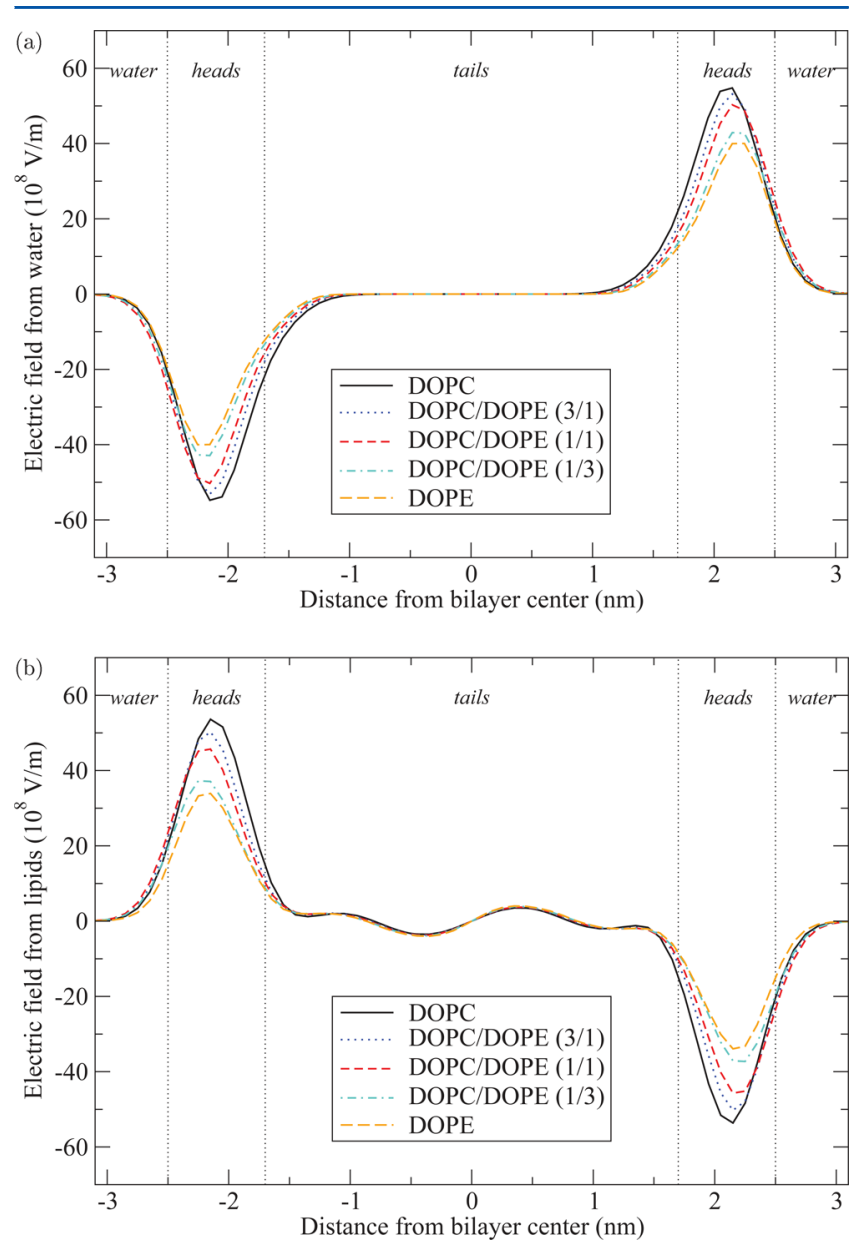

Figure 9. Separated contributions of the electric field from water (a) and lipid molecules (b). Vertical dotted lines indicate approximate boundaries between regions occupied predominantly by water, lipid heads, and hydrocarbon tails.

it can be noticed in general that the water and lipid profiles display extremely large and mostly opposing field strengths, whose main peaks are all centered inside the lipid heads region. The water contribution shows a negative peak on the left-hand side of the profile, and an antisymmetric positive peak on the right-hand side. These peaks originate from a preferential orientation of the water molecular dipoles, whereby the positive ends ( $\mathrm{H}$ atoms) tend to point toward the bilayer center, while the negative ends ( $\mathrm{O}$ atoms) tend to point away from the membrane and toward the bulk water phase. The water electric field is countered by that originating in the lipid molecules; specifically, the lipid headgroup dipoles preferentially point away from the membrane and toward the outer water phase. Inside the hydrocarbon tails region, from -1 to $+1 \mathrm{~nm}$ about the bilayer center, the water contribution is zero, due to the absence of water in the hydrocarbon core. The lipid profile exhibits instead shallow peaks (evidently much smaller in magnitude than those in the heads region), arising from the preferential orientation of dipolar molecular segments along the hydrocarbon tails. In terms of the influence of changes in the lamellar/nonlamellar lipid composition, the presence of increasing amounts of DOPE induces increasing attenuations 
of the magnitude of the peak strength in the heads region, for both the water (Figure 9a) and lipids (Figure 9b) profiles. By comparing the individual profiles with the total $E(z)$ curve (Figure 8), it is clear that the net electric field originates from a nontrivial interplay between the separate contributions from the lipid and water molecules, with large cancellations of enormous opposing field strengths. In fact, the $E(z)$ range for the individual contributions is $-60 \times 10^{8}$ to $+60 \times 10^{8} \mathrm{~V} / \mathrm{m}$, which is an order of magnitude larger than the range of values observed for the net profiles.

\section{CONCLUSIONS}

This study presented a computational investigation into how a number of physical properties of lipid bilayer membranes are affected by changes in the lipid composition. Specifically, we conducted atomistic molecular dynamics simulations of bilayer systems containing the lamellar lipid DOPC and the nonlamellar lipid DOPE in varying proportions. We investigated basic structural properties, as well as depth-dependent distributions of internal pressure, dipole potential, and electric field.

For most of the properties studied, we observed changes proportionally related to the changes in the lipid composition, as intuitively expected. However, the actual amount of change in the physical properties was shown to depend dramatically on the specific properties under investigation. In particular, while structural properties varied only marginally across the different systems, substantial composition-dependent changes were observed for the lateral pressure, dipole potential, and electric field profiles. Increasing the concentration of DOPE was shown to induce a transfer of pressure from the lipid headgroups to the inner hydrocarbon core. Specifically, we observed a decrease in integrated contributions by up to $50 \%$ in the headgroup region and an increase by up to $400 \%$ in the hydrocarbon tail region, when comparing pure DOPC and DOPE systems. The dipole potential profile across the whole membrane was enhanced by the addition of DOPE, with increases of over $20 \%$ for the pure DOPE system compared to pure DOPC. Regarding the electric field, composition changes brought about nontrivial effects in a number of features, including a shift of the main peaks by $0.6 \mathrm{~nm}$ toward the outside of the bilayer, as well as a drop of $41 \%$ in strength at the heads-tails interface.

In summary, we have shown quantitatively that changes in the lamellar vs nonlamellar lipid composition amplify mechanical and electrical signals without significantly altering structural features. The predicted effects on lateral pressure, dipole potential, and electric field profiles are expected to be large enough to affect a range of biological phenomena, including membrane permeation and binding, as well as conformational changes within membrane proteins.

\section{ASSOCIATED CONTENT}

\section{S Supporting Information}

The Supporting Information is available free of charge on the ACS Publications website at DOI: 10.1021/acs.jpcb.5b06604.

Block averaging error estimation analysis, numerical data of structural properties, least-squares fitting results, raw (unsymmetrized) transmembrane profiles, and DOPE inverse phase formation(PDF)

\section{AUTHOR INFORMATION}

\section{Corresponding Author}

*(M.O.) E-mail: m.orsi@qmul.ac.uk.

\section{Notes}

The authors declare no competing financial interest.

\section{ACKNOWLEDGMENTS}

We thank the MidPlus regional centre of excellence (EPSRC grant EP/K000128/1) and the High-End Computing Consortium for Biomolecular Simulation (HECBioSim, EPSRC grant EP/L000253/1) for the provision of computational facilities. W.D. thanks the China Scholarship Council for financial support.

\section{REFERENCES}

(1) Mouritsen, O. G. Life - As a Matter of Fat. The Emerging Science of Lipidomics, 1st ed.; Springer: Berlin, 2005.

(2) Sackmann, E. Supported membranes: scientific and practical applications. Science 1996, 271, 43-48.

(3) Castellana, E. T.; Cremer, P. S. Solid supported lipid bilayers: From biophysical studies to sensor design. Surf. Sci. Rep. 2006, 61, 429-444.

(4) Prausnitz, M. R.; Langer, R. Transdermal drug delivery. Nat. Biotechnol. 2008, 26, 1261-1268.

(5) Orsi, M.; Essex, J. W. Permeability of drugs and hormones through a lipid bilayer: insights from dual-resolution molecular dynamics. Soft Matter 2010, 6, 3797-3808.

(6) Martin, L. J.; Chao, R.; Corry, B. Molecular dynamics simulation of the partitioning of benzocaine and phenytoin into a lipid bilayer. Biophys. Chem. 2014, 185, 98-107.

(7) Lyubartsev, A. P.; Rabinovich, A. L. Recent development in computer simulations of lipid bilayers. Soft Matter 2011, 7, 25-39.

(8) Orsi, M.; Sanderson, W.; Essex, J. W. In Molecular Interactions Bringing Chemistry to Life; Hicks, M. G., Kettner, C., Eds.; BeilsteinInstitut: Frankfurt, Germany, 2007; pp 185-205.

(9) Piggot, T. J.; Pineiro, A.; Khalid, S. Molecular dynamics simulations of phosphatidylcholine membranes: a comparative force field study. J. Chem. Theory Comput. 2012, 8, 4593-4609.

(10) Orsi, M.; Essex, J. W. In Molecular Simulations and Biomembranes: From Biophysics to Function; Sansom, M. S. P., Biggin, P. C., Eds.; The Royal Society of Chemistry: London, 2010; pp 76-90.

(11) Khalid, S.; Bond, P. In Biomolecular Simulations; Monticelli, L., Salonen, E., Eds.; Methods in Molecular Biology; Humana Press: New York, 2013; Vol. 924; pp 635-657.

(12) Humphrey, W.; Dalke, A.; Schulten, K. VMD: visual molecular dynamics. J. Mol. Graphics 1996, 14, 33-38.

(13) Gawrisch, K.; Parsegian, V. A.; Hajduk, D. A.; Tate, M. W.; Gruner, S. M.; Fuller, N. L.; Rand, R. P. Energetics of a hexagonallamellar-hexagonal-phase transition sequence in dioleoylphosphatidylethanolamine membranes. Biochemistry 1992, 31, 2856-2864.

(14) Marsh, D. Lateral pressure profile, spontaneous curvature frustration, and the incorporation and conformation of proteins in membranes. Biophys. J. 2007, 93, 3884-3899.

(15) van den Brink-van der Laan, E.; Killian, J. A.; de Kruijff, B. Nonbilayer lipids affect peripheral and integral membrane proteins via changes in the lateral pressure profile. Biochim. Biophys. Acta, Biomembr. 2004, 1666, 275-88.

(16) Hague, C. V.; Postle, A. D.; Attard, G. S.; Dymond, M. K. Cell cycle dependent changes in membrane stored curvature elastic energy: evidence from lipidomic studies. Faraday Discuss. 2013, 161, 481-497.

(17) Curran, A. R.; Templer, R. H.; Booth, P. J. Modulation of folding and assembly of the membrane protein bacteriorhodopsin by intermolecular forces within the lipid bilayer. Biochemistry 1999, 38, 9328-9336.

(18) Attard, G. S.; Templer, R. H.; Smith, W. S.; Hunt, A. N.; Jackowski, S. Modulation of CTP: phosphocholine cytidylyltransferase 
by membrane curvature elastic stress. Proc. Natl. Acad. Sci. U. S. A. 2000, 97, 9032-9036.

(19) Marsh, D. Protein modulation of lipids, and vice-versa, in membranes. Biochim. Biophys. Acta, Biomembr. 2008, 1778, 15451575.

(20) Manno, S.; Takakuwa, Y.; Mohandas, N. Identification of a functional role for lipid asymmetry in biological membranes: Phosphatidylserine-skeletal protein interactions modulate membrane stability. Proc. Natl. Acad. Sci. U. S. A. 2002, 99, 1943-1948.

(21) Gurtovenko, A. A.; Vattulainen, I. Membrane potential and electrostatics of phospholipid bilayers with asymmetric transmembrane distribution of anionic lipids. J. Phys. Chem. B 2008, 112, 4629-4634.

(22) Cevc, G.; Marsh, D. Phospholipid Bilayers - Physical Principles and Models, 1st ed.; John Wiley \& Sons: New York, 1987.

(23) Templer, R. H.; Castle, S. J.; Curran, A. R.; Rumbles, G.; Klug, D. R. Sensing isothermal changes in the lateral pressure in model membranes using di-pyrenyl phosphatidylcholine. Faraday Discuss. 1999, 111, 41-53.

(24) Ollila, O. H. S.; Vattulainen, I. In Molecular Simulations and Biomembranes: From Biophysics to Function; Sansom, M. S. P., Biggin, P. C., Eds.; The Royal Society of Chemistry: London, 2010; pp 26-55.

(25) Orsi, M.; Noro, M. G.; Essex, J. W. Dual-resolution molecular dynamics simulation of antimicrobials in biomembranes. J. R. Soc., Interface 2011, 8, 826-841.

(26) Cantor, R. S. Lateral pressures in cell membranes: a mechanism for modulation of protein function. J. Phys. Chem. B 1997, 101, 17231725 .

(27) Cantor, R. S. The lateral pressure profile in membranes: a physical mechanism of general anesthesia. Biochemistry 1997, 36, 2339-2344.

(28) Patra, M. Lateral pressure profiles in cholesterol-DPPC bilayers. Eur. Biophys. J. 2005, 35, 79-88.

(29) Ollila, S.; Hyvönen, M. T.; Vattulainen, I. Polyunsaturation in lipid membranes: dynamic properties and lateral pressure profiles. $J$. Phys. Chem. B 2007, 111, 3139-3150.

(30) Orsi, M.; Essex, J. W. Physical properties of mixed bilayers containing lamellar and nonlamellar lipids: insights from coarse-grain molecular dynamics simulations. Faraday Discuss. 2013, 161, 249-272.

(31) Perrin, B. S., Jr.; Sodt, A. J.; Cotten, M. L.; Pastor, R. W. The curvature induction of surface-bound antimicrobial peptides piscidin 1 and piscidin 3 varies with lipid chain length. J. Membr. Biol. 2015, 248, 455-467.

(32) Clarke, R. J. The dipole potential of phospholipid membranes and methods for its detection. Adv. Colloid Interface Sci. 2001, 89-90, 263-281.

(33) Liberman, E.; Topaly, V. Permeability of bimolecular phospholipid membranes for fat-soluble ions. Biofizika 1968, 14, $452-461$.

(34) Starke-Peterkovic, T.; Turner, N.; Else, P. L.; Clarke, R. J. Electric field strength of membrane lipids from vertebrate species: membrane lipid composition and $\mathrm{Na}^{+}-\mathrm{K}^{+}$-ATPase molecular activity. Am. J. Physiol. Regul. Integr. Comp. Physiol. 2005, 288, R663-R670.

(35) Starke-Peterkovic, T.; Clarke, R. J. Effect of headgroup on the dipole potential of phospholipid vesicles. Eur. Biophys. J. 2009, 39, 103-110.

(36) Shinoda, K.; Shinoda, W.; Baba, T.; Mikami, M. Comparative molecular dynamics study of ether-and ester-linked phospholipid bilayers. J. Chem. Phys. 2004, 121, 9648-9654.

(37) Orsi, M.; Michel, J.; Essex, J. W. Coarse-grain modelling of DMPC and DOPC lipid bilayers. J. Phys.: Condens. Matter 2010, 22, 155106.

(38) Pickar, A. D.; Benz, R. Transport of oppositely charged lipophilic probe ions in lipid bilayer membranes having various structures. J. Membr. Biol. 1978, 44, 353-376.

(39) Schamberger, J.; Clarke, R. J. Hydrophobic ion hydration and the magnitude of the dipole potential. Biophys. J. 2002, 82, 30813088.

(40) Nagle, J. F.; Tristram-Nagle, S. Structure of lipid bilayers. Biochim. Biophys. Acta, Rev. Biomembr. 2000, 1469, 159-195.
(41) Sodt, A. J.; Pastor, R. W. Bending free energy from simulation: correspondence of planar and inverse hexagonal lipid phases. Biophys. J. 2013, 104, 2202-2211.

(42) Venable, R. M.; Sodt, A. J.; Rogaski, B.; Rui, H.; Hatcher, E.; MacKerell, A. D., Jr; Pastor, R. W.; Klauda, J. B. CHARMM all-atom additive force field for sphingomyelin: elucidation of hydrogen bonding and of positive curvature. Biophys. J. 2014, 107, 134-145.

(43) Sodt, A. J.; Pastor, R. W. Molecular modeling of lipid membrane curvature induction by a peptide: more than simply shape. Biophys. J. 2014, 106, 1958-1969.

(44) Martínez, L.; Andrade, R.; Birgin, E. G.; Martínez, J. M. Packmol: A package for building initial configurations for molecular dynamics simulations. J. Comput. Chem. 2009, 30, 2157-2164.

(45) Klauda, J. B.; Venable, R. M.; Freites, J. A.; O'Connor, J. W.; Tobias, D. J.; Mondragon-Ramirez, C.; Vorobyov, I.; MacKerell, A. D., Jr; Pastor, R. W. Update of the CHARMM all-atom additive force field for lipids: validation on six lipid types. J. Phys. Chem. B 2010, 114, $7830-7843$

(46) Brooks, B. R.; Bruccoleri, R. E.; Olafson, B. D.; States, D. J.; Swaminathan, S.; Karplus, M. CHARMM: A program for macromolecular energy, minimization, and dynamics calculations. J. Comput. Chem. 1983, 4, 187-217.

(47) Durell, S. R.; Brooks, B. R; Ben-Naim, A. Solvent-induced forces between two hydrophilic groups. J. Phys. Chem. 1994, 98, 21982202.

(48) Plimpton, S. Fast parallel algorithms for short-range molecular dynamics. J. Comput. Phys. 1995, 117, 1-19.

(49) LAMMPS website, http://lammps.sandia.gov, accessed July 9, 2015.

(50) Schneider, T.; Stoll, E. Molecular-dynamics study of a threedimensional one-component model for distortive phase transitions. Phys. Rev. B: Condens. Matter Mater. Phys. 1978, 17, 1302.

(51) Berendsen, H. J.; Postma, J. P. M.; van Gunsteren, W. F.; DiNola, A.; Haak, J. Molecular dynamics with coupling to an external bath. J. Chem. Phys. 1984, 81, 3684-3690.

(52) Harvey, S. C.; Tan, R. K. Z.; Cheatham, T. E., III The flying ice cube: Velocity rescaling in molecular dynamics leads to violation of energy equipartition. J. Comput. Chem. 1998, 19, 726-740.

(53) MacKerell, A. D., Jr.; Bashford, D.; Bellott, M.; Dunbrack, R., Jr.; Evanseck, J.; Field, M.; Fischer, S.; Gao, J.; Guo, H.; Ha, S.; et al. Allatom empirical potential for molecular modeling and dynamics studies of proteins. J. Phys. Chem. B 1998, 102, 3586-3616.

(54) Hockney, R. W.; Eastwood, J. W. Computer Simulation Using Particles; Adam Hilger: New York, 1989.

(55) Orsi, M. Comparative assessment of the ELBA coarse-grained model for water. Mol. Phys. 2014, 112, 1566-1576.

(56) Flyvbjerg, H.; Petersen, H. G. Error estimates on averages of correlated data. J. Chem. Phys. 1989, 91, 461-466.

(57) Rapaport, D. C. The Art of Molecular Dynamics Simulation, 2nd ed.; Cambridge University Press: Cambridge, U.K., 2004.

(58) Orsi, M.; Haubertin, D. Y.; Sanderson, W. E.; Essex, J. W. A quantitative coarse-grain model for lipid bilayers. J. Phys. Chem. B 2008, 112, 802-815.

(59) Varnik, F.; Baschnagel, J.; Binder, K. Molecular dynamics results on the pressure tensor of polymer films. J. Chem. Phys. 2000, 113, 4444-4453.

(60) Harasima, A. Molecular theory of surface tension. Adv. Chem. Phys. 1958, 1, 203-237.

(61) Orsi, M.; Essex, J. W. The ELBA force field for coarse-grain modeling of lipid membranes. PLoS One 2011, 6, e28637.

(62) Kučerka, N.; Nagle, J. F.; Sachs, J. N.; Feller, S. E.; Pencer, J.; Jackson, A.; Katsaras, J. Lipid bilayer structure determined by the simultaneous analysis of neutron and X-ray scattering data. Biophys. J. 2008, 95, 2356-2367.

(63) Kučerka, N.; Tristram-Nagle, S.; Nagle, J. Structure of fully hydrated fluid phase lipid bilayers with monounsaturated chains. J. Membr. Biol. 2006, 208, 193-202.

(64) Pan, J.; Tristram-Nagle, S.; Kučerka, N.; Nagle, J. F. Temperature dependence of structure, bending rigidity, and bilayer 
interactions of dioleoylphosphatidylcholine bilayers. Biophys. J. 2008, 94, 117-124.

(65) Rand, R.; Parsegian, V. Hydration forces between phospholipid bilayers. Biochim. Biophys. Acta, Rev. Biomembr. 1989, 988, 351-376.

(66) Rand, R.; Fuller, N.; Gruner, S.; Parsegian, V. Membrane curvature, lipid segregation, and structural transitions for phospholipids under dual-solvent stress. Biochemistry 1990, 29, 76-87.

(67) Greenwood, A. I.; Tristram-Nagle, S.; Nagle, J. F. Partial molecular volumes of lipids and cholesterol. Chem. Phys. Lipids 2006, $143,1-10$.

(68) Rand, R; Fuller, N. Structural dimensions and their changes in a reentrant hexagonal-lamellar transition of phospholipids. Biophys. J. 1994, 66, 2127.

(69) Chen, Z.; Rand, R. The influence of cholesterol on phospholipid membrane curvature and bending elasticity. Biophys. J. 1997, 73, 267.

(70) Gruner, S. M.; Tate, M.; Kirk, G.; So, P.; Turner, D.; Keane, D.; Tilcock, C.; Cullis, P. X-ray diffraction study of the polymorphic behavior of $\mathrm{N}$-methylated dioleoylphosphatidylethanolamine. Biochemistry 1988, 27, 2853-2866.

(71) Reddy, A. S.; Warshaviak, D. T.; Chachisvilis, M. Effect of membrane tension on the physical properties of DOPC lipid bilayer membrane. Biochim. Biophys. Acta, Biomembr. 2012, 1818, 2271-2281.

(72) Levine, Z. A.; Venable, R. M.; Watson, M. C.; Lerner, M. G.; Shea, J.-E.; Pastor, R. W.; Brown, F. L. H. Determination of biomembrane bending moduli in fully atomistic simulations. J. Am. Chem. Soc. 2014, 136, 13582-13585.

(73) Raghunathan, M.; Zubovski, Y.; Venable, R. M.; Pastor, R. W.; Nagle, J. F.; Tristram-Nagle, S. Structure and elasticity of lipid membranes with genistein and daidzein bioflavinoids using X-ray scattering and MD simulations. J. Phys. Chem. B 2012, 116, 39183927.

(74) Warshaviak, D. T.; Muellner, M. J.; Chachisvilis, M. Effect of membrane tension on the electric field and dipole potential of lipid bilayer membrane. Biochim. Biophys. Acta, Biomembr. 2011, 1808, $2608-2617$.

(75) de Vries, A. H.; Mark, A. E.; Marrink, S. J. The binary mixing behavior of phospholipids in a bilayer: a molecular dynamics study. $J$. Phys. Chem. B 2004, 108, 2454-2463.

(76) Ben-Shaul, A. In Structure and Dynamics of Membranes; Lipowsky, R., Sackmann, E., Eds.; Elsevier: Amsterdam, 1995; pp 359-401.

(77) Mukhin, S. I.; Baoukina, S. Analytical derivation of thermodynamic characteristics of lipid bilayer from a flexible string model. Phys. Rev. E 2005, 71, 061918.

(78) Polyansky, A. A.; Volynsky, P. E.; Nolde, D. E.; Arseniev, A. S.; Efremov, R. G. Role of lipid charge in organization of water/lipid bilayer interface: Insights via computer simulations. J. Phys. Chem. B 2005, 109, 15052-15059.

(79) Siu, S. W. I.; Vacha, R; Jungwirth, P.; Bockmann, R. A. Biomolecular simulations of membranes: Physical properties from different force fields. J. Chem. Phys. 2008, 128, 125103.

(80) Vorobyov, I.; Allen, T. W. The electrostatics of solvent and membrane interfaces and the role of electronic polarizability. J. Chem. Phys. 2010, 132, 185101.

(81) Orsi, M.; Ding, W.; Palaiokostas, M. Direct mixing of atomistic solutes and coarse-grained water. J. Chem. Theory Comput. 2014, 10, 4684-4693.

(82) Ding, W.; Palaiokostas, M.; Orsi, M. Stress testing the ELBA water model. Mol. Simul. 2016, 42, 337-346.

(83) Shrestha, R.; Cardenas, A. E.; Elber, R.; Webb, L. J. Measurement of the membrane dipole electric field in DMPC vesicles using vibrational shifts of p-cyanophenylalanine and molecular dynamics simulations. J. Phys. Chem. B 2015, 119, 2869-2876. 